\title{
Backward figural masking as a function of intercontour distance
}

HELEN W. STREICHER AND ROBERT H. POLLACK

INSTITUTE FOR JUVENILE RESEARCH, CHICAGO

The intervals over which a ring whose inner diameter subtended 30'visual angle masked disk test figures of 10,15 , 20 , and 25' and a square test figure with 20' sides were obtained for two $S s$. In the case of parallel contours, masking increased as intercontour distance decreased. For the 10' disk and the square, masking was negligible. The data were discussed with regard to the apparent "facilitation" effect found in forward masking when a large masking disk is followed by a small disk test figure.

In a recent study of forward visual masking, Sturr, Frumkes, \& Veneruso (1965) investigated the effect of varying the size of a preadapting masking figure (MF) on the time to perceive a smaller test figure (TF). Using a round $10^{\prime} \mathrm{TF}$ and round MFs of $15,22.5$, and $30^{\prime}$, they found, as anticipated, that time to detect TF, relative to "resting threshold" decreased as MF size increased, both in the fovea and in the periphery. One finding which was not anticipated was that, in the fovea, preadaptation to the $30^{\prime} \mathrm{MF}$ produced, not masking, but facilitation-the threshold for detection of $T F$ was, in this instance, lower than the resting threshold obtained when no MF was presented.

A probable explanation for the "facilitation" follows from analysis of their procedure. Their resting threshold was the time to detect a black TF on a gray ground after preadaptation to a white field. In the evaluation of the effect of MF size on time to detect TF, however, Ss were preadapted to the black MF on white ground. Preadaptation to $M F$ in this way probably produced an afterimage approximating the size of MF on the gray ground on which TF was then presented, a relatively bright afterimage whose contours were more or less close to those of $\mathrm{TF}$, depending on the size of MF. When MF was $15^{\prime}$ and TF $10^{\prime}$, there should have been an attraction of the parallel contours of the MF afterimage and TF (Pollack, 1963), in effect brightening $T F$ and producing an elevated threshold (masking). If there were little or no interaction between contours of a $30^{\prime}$ afterimage and a $10^{\prime} \mathrm{TF}$, then the threshold for detection of TF would have been lowered due to its having been presented on a brighter immediate ground (the afterimage) than that available during measurement of the resting threshold. Therefore, the apparent facilitation of detection of $\mathrm{TF}$ by presentation of a relatively large $\mathrm{MF}$ may have been due simply to the lack of interaction between the contour of the small TF and that of the afterimage of the large $\mathrm{MF}$.

Data on the effect of increasing intercontour distance on figural masking were necessary for support of the above analysis of the "facilitation" of TF by MF. This study was designed to provide those data, along with data for figures whose contours are close to each other but not parallel. In order to avoid the confounding influence of afterimages, the backward figural masking (TF presented before MF) design was used. Subjects

The authors, both in their thirties, each served as E and S.

\section{Apparatus}

A three-channel tachistoscope, Scientific Prototype Model GA, was used. Intensities of all channels were adjusted to provide Macbeth Illuminometer readings of $5.0 \mathrm{ft} .-\mathrm{c}$. from the background used in the study. Procedure

The MF, mounted on black ground, was a white ring, 5 ' thick, whose inner diameter subtended a visual angle of $30^{\prime}$, outer diameter $40^{\prime}$. The TFs were midgray disks, also on black ground, with diameters of $10,15,20$, and 25'. The distances between the inner contour of MF and the outer contours of TFs were therefore $10.0,7.5,5.0$, and 2.5', respectively. One additional TF, a square with $20^{\prime}$ sides, was used.

TF and MF were each presented for $15 \mathrm{msec}$., separated by an interval varied in $10-\mathrm{msec}$. steps during which only the background was present. For each $\mathbf{T F}$, the interstimulus interval necessary for detection of TF (the masking interval) was obtained for both Ss using the method of limits, 32 runs, eight ascending and eight descending series in each of two sessions. Sessions were always run at the same time of day.

\section{Results and Discussion}

The data for both Ss are presented in Fig. 1. From these data it is clear that there is a negative relationship between masking and intercontour distance when contours are parallel. Differences between the mean masking intervals for the disk TFs were highly significant for both RHP ( $F=78.77, d f=3 / 124)$ and HWS $(\mathrm{F}=125.40, \mathrm{df}=3 / 124)$. In a test for trend, only the linear component was significant for RHP ( $F=232.84$, $\mathrm{df}=1 / 124)$. For HWS, both linear regression $(\mathrm{F}=$ 301.65, $\mathrm{df}=1 / 124)$ and the quadratic component $(\mathrm{F}=$ 37.27, $\mathrm{df}=2 / 124$ ) were significant beyond the .001 level of confidence. The obtained masking intervals were somewhat low overall, this probably being due to the relatively advanced age (perceptually, that is) of the Ss (Pollack, 1965b).

Masking data for the square TF were plotted between 2.5 and $5.0^{\prime}$, since at the midpoint of a side its contour was $5.0^{\circ}$ from the inner contour of $\mathrm{MF}$, 


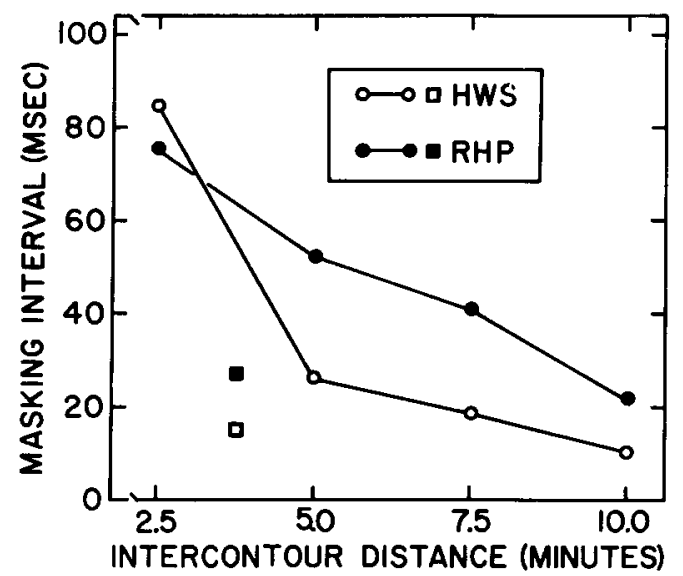

Fig. 1. Mean interstimulus interval for backward figural masking as a function of intercontour distance. Circles indicate disk TFs; squares indicate square TF.

but at a corner, only about $2.5^{\prime}$. This $\mathrm{TF}$, although intermediate in intercontour distance between the two largest disk TFs, was masked considerably less than either of them. In fact, the mean masking interval for the square was not significantly different from that for the smallest disk for either S. These data are in accord with earlier results (Pollack, 1965a) showing that when contours of TF are not parallel to those of MF, much less masking occurs than when they are parallel, even if intercontour distances are comparable.

No facilitation of detection of a small TF using a large $M F$ was found in this situation. The relatively small amount of masking produced when the separation between the inner contour of the ring and the outer contour of the disk makes it appear quite likely that the facilitation reported by Sturr, Frumkes, \& Veneruso (1965) was an artifact of an experimental design subject to the production of afterimages, afterimages which, when they were large relative to $\mathrm{TF}$, could increase the contrast between TF and the immediate ground over the contrast present during testing for the resting threshold. Further research is being conducted in order to provide additional support for this conclusion.

\section{References}

Pollack, R. H. Effects of temporal order of stimulus presentation on the direction of figural aftereffects. Percept. mot. Skills, $1963,17,875-880$.

Pollack, R. H. Effects of figure-ground contrast and contour orientation on figural masking. Psychon. Sci., 1965a, 2, 369-370.

Pollack, R. H. Backward figural masking as a function of chronological age and intelligence. Psychon. Sci., 1965b, 3, 65-66. Sturr, J. H., Frumkes, T. E., \& Veneruso, D. M. Spatial determinants of visual masking: Effects of mask size and retinal position. Psychon. Sci., 1965, 3, 327-328.

\section{Note}

1. This research was partially supported by Grant No. HD 01433 awarded by the National Institute of Child Health and Human Development. 